\title{
Asymmetric Barrier Lyapunov Function-Based Wheel Slip Control for Antilock Braking System
}

\author{
Xiaolei Chen, Zhiyong Dai, Hui Lin, Yanan Qiu, and Xiaogeng Liang \\ School of Automation, Northwestern Polytechnical University, Xian, Shaanxi 710129, China \\ Correspondence should be addressed to Zhiyong Dai; daizhiyong_nwpu@hotmail.com
}

Received 2 May 2015; Accepted 21 July 2015

Academic Editor: Ronald M. Barrett

Copyright ( 2015 Xiaolei Chen et al. This is an open access article distributed under the Creative Commons Attribution License, which permits unrestricted use, distribution, and reproduction in any medium, provided the original work is properly cited.

\begin{abstract}
As an important device of the aircraft landing system, the antilock braking system (ABS) has a function to avoid aircraft wheels selflocking. To deal with the strong nonlinear characteristics, complex nonlinear control schemes are applied in ABS. However, none of existing control schemes focus on the braking operating status, which directly reflects wheels self-locking degree. In this paper, the braking operating status region is divided into three regions: the healthy region, the light slip region, and the deep slip region. An ABLF-based wheel slip controller is proposed for ABS to constrain the braking system operating status in the healthy region and the light slip region. Therefore the ABS will be prevented from operating in the deep slip region. Under the proposed control scheme, self-locking is avoided completely and zero steady state error tracking of the wheel optimal slip ratio is implemented. The Hardware-In-Loop (HIL) experiments have validated the effectiveness of the proposed controller.
\end{abstract}

\section{Introduction}

Antilock braking system (ABS), as an important safety guarantee of aircraft landing process, which keeps the braking direction stable and enhances braking performance, has a function to control aircraft landing dynamics. With the help of ABS, self-locking of main wheels and lost direction of front wheels, which will give rise to the sideslip and tail, are avoided. Meanwhile, under a good performance of ABS control, stopping distance is shortened, stopping time is saved, and tire wear is also reduced.

Taking advantage that the wheel speed is easy to obtain and the control scheme is simple to realize, the traditional ABS control is based on the wheel deceleration rate. Compared with the traditional control, ABS based on the wheel slip ratio control has a better control performance $[1,2]$. The main objective of the slip control is to regulate wheel slip at its optimum value, which ensures that the aircraft braking system has a maximum tire-road friction. When the wheel slip of ABS operates at the optimum value, the wheel self-locking is avoided. Based on wheel slip control, some solutions have been proposed to deal with this complex nonlinear problem, such as neural network control [3], sliding mode control $[1,2,4-6]$, integral feedback and back stepping control [7], combined control [8-10], iterative learning control [11], fuzzy control $[12,13]$, adaptive control $[10,14]$, nonlinear control $[7,15,16]$, and extremum seeking control [17-19]. These studies always focus on how to track the optimum slip ratio with a good dynamic performance and steady state accuracy. However, none of the previously mentioned schemes take into account braking operating status, which directly reflects wheel slip degree.

In fact, in the slip ratio control, the braking operating region can be divided into three regions (the healthy region, the light slip region, and the deep slip region) dependent on the relationship between the slip ratio and the combination coefficient [20]. When the ABS operates in the deep slip region, not only is the system unable to obtain the maximum tire-road friction, but also the tires would be self-locking finally. Obviously, the ABS control should be designed to avoid braking operating status in the deep slip region.

In practice, output-constrained tracking control is an important problem to deal with and some results have been achieved. Among them, [21-24] propose Barrier Lyapunov Function- (BLF-) based constrained control, which has a good tracking performance without violation of any constraint. Taking into account the initial condition of aircraft 
landing process, an Asymmetric Barrier Lyapunov Function(ABLF-) based ABS wheel slip control scheme is proposed to constrain braking system operating status in the healthy region and the light slip region rather than the deep slip region. It prevents wheels from self-locking. In addition, the wheel slip ratio tracks optimum slip ratio with a zero steady state error.

The paper is organized as follows: Section 2 describes aircraft landing system. Section 3 formulates the Barrier Lyapunov Function problem. And Section 4 designs the controller based on ABLF for antilock braking. Section 5 analyzes HIL experiment results. Section 6 contains concluding remarks.

\section{System Dynamics}

Establishing the aircraft landing system model is the basis of the ABS controller design. Compared with common vehicles models, the aircraft landing system is affected by many aerodynamic forces, such as aerodynamic drag, aerodynamic lift, and parachute drag, which all are nonlinear parts with respect to aircraft speed. It makes the aircraft ABS model more complex and difficult to control. In order to simplify system model, some assumptions are as follows:

(i) The aircraft is ideal rigid one.

(ii) In the aircraft braking process, the engine is at idle state or reverse state.

(iii) The system ignores the crosswind effect.

(iv) The system ignores the tire deformation.

(v) All wheels performances are the same, and they are controlled synchronously.

The aircraft force analysis is shown in Figure 1 and the parameters employed in the system are given in Table 1. The force balance equation of aircraft landing system is

$$
\begin{array}{r}
m \dot{V}_{x}-T_{0}+F_{x}+F_{s}+n F_{f}=0, \\
F_{y}+N_{2}+n N_{1}-m g_{a}=0, \\
N_{2} b-n F_{f} h_{c}-n N_{1} a+F_{s} h_{s}-T_{0} h_{t}=0 .
\end{array}
$$

According to the aerodynamic theory, we have aerodynamic force functions as (2) and the definition of the coefficients is described in Table 2:

$$
\begin{aligned}
& F_{x}=\frac{1}{2} \rho C_{x} S_{x} V_{x}^{2}, \\
& F_{y}=\frac{1}{2} \rho C_{y} S_{y} V_{x}^{2}, \\
& F_{s}=\frac{1}{2} \rho C_{s x} S_{s x} V_{x}^{2}, \\
& T_{0}=T_{0 \text { _ini }}+k_{t} V_{x} .
\end{aligned}
$$

\begin{tabular}{|c|c|c|}
\hline Name & Description & Value \\
\hline$V_{x}$ & Aircraft speed & \\
\hline$T_{0}$ & Engine force in idle state & \\
\hline$F_{x}$ & Aerodynamic drag & \\
\hline$F_{y}$ & Aerodynamic lift & \\
\hline$F_{s}$ & Parachute drag & \\
\hline$F_{f}$ & Braking friction force between tire and ground & \\
\hline$N_{1}$ & Main wheel support force & \\
\hline$N_{2}$ & Front wheel support force & \\
\hline$n$ & Number of main wheels & 4 \\
\hline$h_{c}$ & Center of gravity height & $2.82 \mathrm{~m}$ \\
\hline$h_{s}$ & $\begin{array}{c}\text { Distance between parachute drag line } \\
\text { and center of gravity }\end{array}$ & $0.8 \mathrm{~m}$ \\
\hline$h_{t}$ & $\begin{array}{l}\text { Distance between engine force line } \\
\text { and center of gravity }\end{array}$ & $0.23 \mathrm{~m}$ \\
\hline$a$ & $\begin{array}{l}\text { Distance between main wheel and center of } \\
\text { gravity }\end{array}$ & $1.52 \mathrm{~m}$ \\
\hline$b$ & $\begin{array}{l}\text { Distance between front wheel and center of } \\
\text { gravity }\end{array}$ & $7.67 \mathrm{~m}$ \\
\hline$g_{a}$ & Gravity acceleration & $9.8 \mathrm{~m} / \mathrm{s}^{2}$ \\
\hline$m$ & Weight of the aircraft & $15 \mathrm{t}$ \\
\hline
\end{tabular}

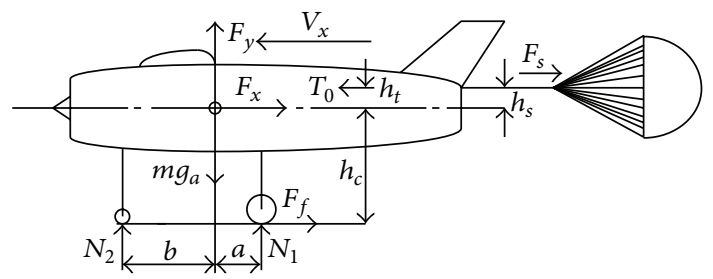

FIgURE 1: Aircraft landing system.

TABLE 1: Parameters for the aircraft model.

TABle 2: Parameters of the coefficients.

\begin{tabular}{lcc}
\hline Name & Description & Value \\
\hline$\rho$ & Air density & 0.155 \\
$C_{y}$ & Aerodynamic lift coefficient & 0.65 \\
$C_{x}$ & Aerodynamic drag coefficient & 0.13 \\
$C_{s x}$ & Parachute drag coefficient & 0.85 \\
$S_{x}$ & Aerodynamic drag area & $38.6 \mathrm{~m}^{2}$ \\
$S_{y}$ & Aerodynamic lift area & $55.6 \mathrm{~m}^{2}$ \\
$S_{s x}$ & Parachute area & $7.5 \mathrm{~m}^{2}$ \\
$k_{t}$ & Velocity coefficient of engine & 1.1 \\
$T_{0 \_ \text {ini }}$ & The intimal engine force in idle state & $553 \mathrm{~N}$ \\
\hline
\end{tabular}

The combination coefficient $\mu(\lambda)$ is defined as

$$
\mu(\lambda)=\frac{F_{f}}{N_{1}} .
$$

Substitute (3) into (1), and we obtain

$$
\dot{V}_{x}=\frac{T_{0}-F_{x}-F_{s}}{m}-\frac{\mu(\lambda)}{m} \frac{m g_{a} b-F_{y} b+F_{s} h_{s}-T_{0} h_{t}}{a+b+\mu(\lambda) h_{c}} .
$$




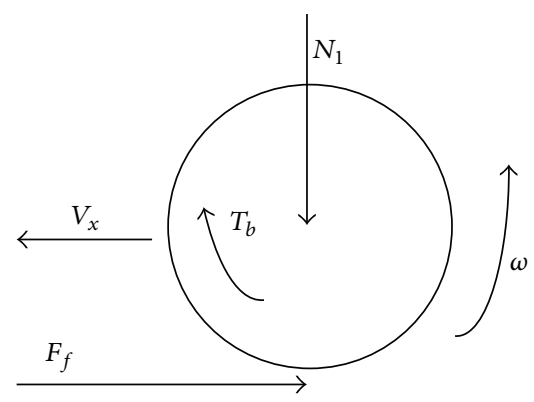

Figure 2: Single wheel model force analysis.

The model of wheel dynamics is given in Figure 2. Taking the main single wheel model into account (shown in Figure 2), the wheel dynamics are described as follows:

$$
I \dot{\omega}=-B \omega+F_{f} R_{g}-T_{b}=-B \omega+N_{1} R_{g} \mu(\lambda)-T_{b},
$$

where $\omega$ is angular velocity of the wheel; $I$ is inertia of the wheel; $N_{1}$ is support force of the wheel (the same as (1)); $R_{g}$ is radius of the wheel; $B$ is drag torque coefficient; $T_{b}$ is braking torque; the wheel slip ratio $\lambda$ is defined as

$$
\lambda=\frac{V_{x}-R_{g} \omega}{V_{x}} .
$$

Since $R_{g} \omega \leq V_{x}$, we have that $0 \leq \lambda \leq 1$ from (6); while $\lambda=$ 0 , braking friction force is zero; and while $\lambda=1$, the wheel is in the self-locking state, which will cause sideslip and tail. There exists optimal slip ratio $\lambda^{*}$. When $\lambda=\lambda^{*}$, the braking system has the maximum friction coefficient $\mu^{*} \cdot \mu$ and $\lambda$ have a nonlinear relationship as follows [25] (shown in Figure 3):

$$
\mu(\lambda)=2 \mu^{*} \frac{\lambda^{*} \lambda}{\lambda^{* 2}+\lambda^{2}} .
$$

As shown in Figure 3(a), braking operating status region is divided into three regions: the healthy region, where
$0 \leq \lambda \leq \lambda^{*}$, the light slip region, where $\lambda^{*}<\lambda \leq k_{c}$, and the deep slip region, where $k_{c}<\lambda \leq 1$. If wheel slip ratio is $\lambda_{1}$ (shown in Figure 3(b)), the braking operating status is in the healthy region. When the braking torque $T_{b}$ is applied to the main wheel, according to (5), the wheel angular velocity $\omega$ decreases. As a result, $\lambda$ increases from $\lambda_{1}$ to $\lambda_{2}$ and $\mu$ increases to $\mu_{2}$, which will give birth to the redaction of $V_{x}$ and increase of $\lambda$ to $\lambda_{3}$. Accordingly, $\mu$ decreases from $\mu_{2}$ to $\mu_{3}$ and the system would be stable finally. If wheel slip is $\lambda_{4}$ (shown in Figure 3(c)), the braking operating status is in the slip region (the light slip region or the deep slip region). When braking torque $T_{b}$ is applied to the main wheel, $\omega$ decreases and $\lambda$ increases from $\lambda_{4}$ to $\lambda_{5}$. Correspondingly, $\mu$ decreases to $\mu_{5}$, which will give birth to the increase of $V_{x}$ and redaction of $\lambda$ to $\lambda_{6}$. As a result, $\mu$ decreases from $\mu_{5}$ to $\mu_{6}$. Finally, $\lambda$ will increase up to one and the wheel will be selflocking. Compared with operating in the light slip region, the braking system operating in the deep slip region will cause more severe tire wear and even the wheel self-locking. It is the reason why the control law should keep ABS working in the healthy region and the light slip region, where $0 \leq \lambda<k_{c}$.

According to (6), we obtain

$$
\dot{\lambda}=\frac{(1-\lambda) \dot{V}_{x}-R_{g} \dot{\omega}}{V_{x}} .
$$

Substituting (4), (5), and (2) into (8), we have

$$
\begin{aligned}
\dot{\lambda} & =\frac{(1-\lambda)}{V_{x}}\left(\frac{T_{0}-F_{x}-F_{s}}{m}\right. \\
& \left.-\frac{\mu(\lambda)}{m} \frac{m g_{a} b-F_{y} b+F_{s} h_{s}-T_{0} h_{t}}{a+b+\mu(\lambda) h_{c}}\right)-\frac{R_{g}}{V_{x} I}(-B \omega \\
& \left.+N_{1} R_{g} \mu(\lambda)-T_{b}\right)=f(\lambda)+\frac{R_{g}}{V_{x} I} T_{b},
\end{aligned}
$$

where

$$
\begin{aligned}
f(\lambda) & =\frac{1-\lambda}{V_{x}}\left(\frac{T_{0}-F_{x}-F_{s}}{m}-\frac{\mu(\lambda)}{m} \frac{m g_{a} b-F_{y} b+F_{s} h_{s}-T_{0} h_{t}}{a+b+\mu(\lambda) h_{c}}\right)-\frac{R_{g}}{V_{x} I}\left(-B \omega+N_{1} R_{g} \mu(\lambda)\right) \\
& =\frac{1-\lambda}{V_{x}}\left[\frac{\left(T_{0_{\mathrm{ini}}}+k_{t} V_{x}\right)-(1 / 2) \rho C_{x} S_{x} V_{x}^{2}-(1 / 2) \rho C_{s x} S_{s x} V_{x}^{2}}{m}\right. \\
& \left.-\frac{\mu(\lambda)}{m} \frac{\left(m g_{a} b-(1 / 2) \rho C_{y} S_{y} V_{x}^{2} b+(1 / 2) \rho C_{s x} S_{s x} V_{x}{ }^{2} h_{s}-\left(T_{0_{\mathrm{ini}}}+k_{t} V_{x}\right) h_{t}\right)}{a+b+\mu(\lambda) h_{c}}\right]-\frac{R_{g}}{V_{x} I}\left(-B \omega+N_{1} R_{g} \mu(\lambda)\right) .
\end{aligned}
$$

From (10), we find that the braking system has a strong nonlinear characteristic which is caused by the following factors:

(i) The relationship of the wheel slip ratio with the wheel speed and the vehicle speed is nonlinear. (ii) The $\mu-\lambda$ relationship is nonlinear.

(iii) There are multiplicative terms in the system state function.

(iv) Equations (4) and (5) are nonlinear. 


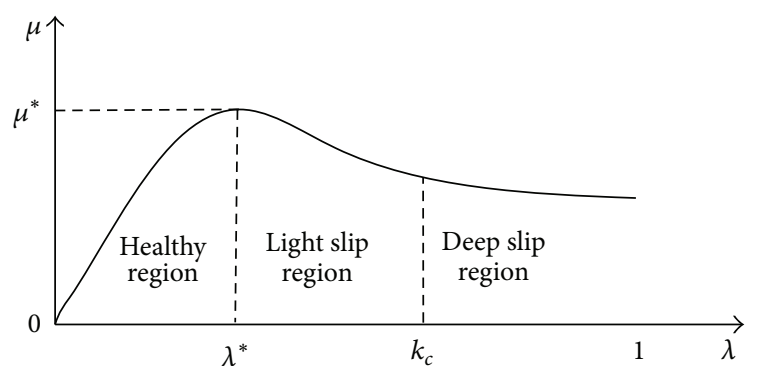

(a) $\mu$ and $\lambda$

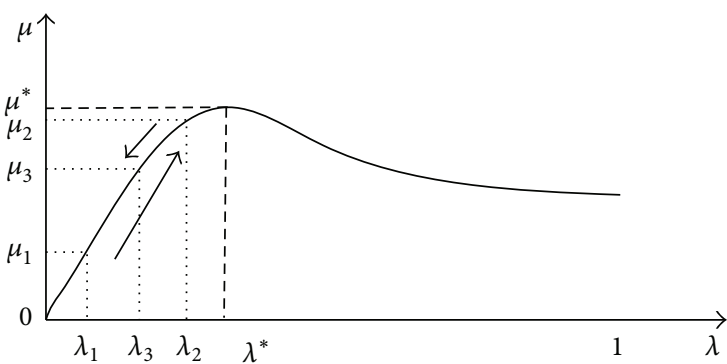

(b) Health region

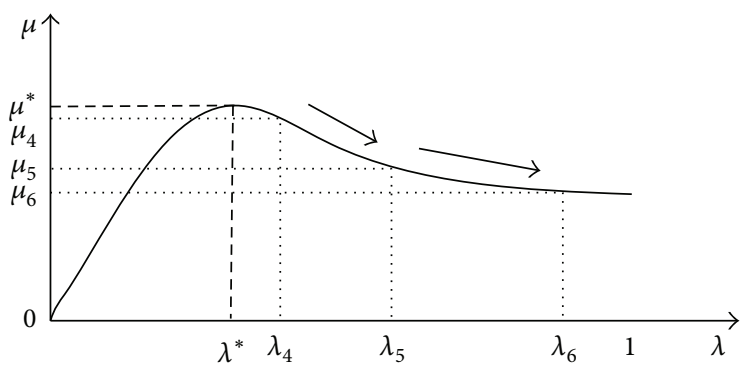

(c) Slip region

FIgURE 3: Relationship between $\mu$ and $\lambda$.

Taking into account the braking operating status region, ABS control problem is described as follows.

If possible, design a nonlinear controller for system (9) such that

(1) the closed loop system is stable;

(2) $\lim _{t \rightarrow \infty}\left|\lambda-\lambda^{*}\right|=0$;

(3) the closed ABS operates in the healthy region and the light slip region. It implies that $0 \leq \lambda<k_{c}$.

\section{Barrier Lyapunov Function Preliminaries}

In practice, it is an important problem for a fixed plant to track a desired trajectory $y_{d}(t)$ while ensuring that all closed loop signals are bounded and that the output constraint is not violated. To prevent the output from violating the constraint, we employ a Barrier Lyapunov Function (BLF), which is defined as follows [24].

Definition 1. Barrier Lyapunov Function is a continuously differentiable and positive definite scalar function $V(x)$, defined with respect to the system

$$
\dot{x}=f(x)
$$

on an open region $\mathscr{D}$ containing the origin. And it has the property $V(x) \rightarrow \infty$ as $x$ approaches the boundary of $\mathscr{D}$ and satisfies $V(x(t)) \leq b, \forall t \geq 0$ along the solution of $\dot{x}=f(x)$ for $x(0) \in \mathscr{D}$ and some positive constant $b$.

A useful lemma [24] is as follows.
Lemma 2. An open set $\mathscr{Z}:=\left\{z \in \mathbb{R}:-k_{a}<z<k_{b}\right\} \subset \mathbb{R}$, where $k_{a}$ and $k_{b}$ are known constants. Consider the system

$$
\dot{z}=h(t, z),
$$

where $h: \mathbb{R}_{+} \times \mathscr{Z} \rightarrow \mathbb{R}$ is piecewise continuous in t and locally Lipschitz in $z$, uniformly in $t$, on $\mathbb{R}_{+} \times \mathscr{Z}$. Suppose there exists a continuously differentiable function $V: \mathscr{Z} \rightarrow \mathbb{R}_{+}$positive definite such that

$$
V(z) \longrightarrow \infty \quad \text { as } z \longrightarrow-k_{a} \text { or } z \longrightarrow k_{b} .
$$

If the initial condition satisfies $z(0) \in\left(-k_{a}, k_{b}\right)$ and $V(z)$ derivative $\dot{V}(z)$ is negative definite, $z(t)$ remains in the open set $z \in\left(-k_{a}, k_{b}\right), \forall t \in[0, \infty)$.

\section{Asymmetric Barrier Lyapunov Function Controller}

In the ABS design, the objective of control scheme is to ensure that the wheel slip ratio $\lambda$ tracks the optimum wheel slip ratio $\lambda^{*}$. In the different road conditions, the optimum wheel slip ratios are not the same. And the optimum wheel slip ratio can be obtained by the identification technology. In this paper, the dry road condition is chosen and the optimum wheel slip ratio $\lambda^{*}$ is 0.25 . To ensure that the wheel slip ratio remains operating in the healthy region and the light slip region (it is $\left.\lambda(t)<k_{c}\right)$, an output-constraint control is designed. Since the initial condition of the aircraft landing system is $\lambda(0)=0$, which does not satisfy the initial condition of BLF function, an Asymmetric Barrier Lyapunov Function [24] (ABLF) is employed to relax the initial condition. 
Considering system (9), the aircraft landing system can be rewritten as

$$
\begin{aligned}
& \dot{x}=f(x)+g(x) u, \\
& y=x
\end{aligned}
$$

where the state variable $x=\lambda$. The input $u=T_{b}$. The function $f(x)$ is defined in (10). And the function $g(x)=$ $R_{g} /\left(V_{x} I\right)$. In the aircraft braking process, when the aircraft speed $V_{x}$ decreases to a fixed speed $V_{x}^{r}$, which is a small positive constant such as $0.1 \mathrm{~m} / \mathrm{s}$, the ABS will be turned off and the aircraft stops by itself. So $g(x)$ is positive, $\forall t \in[0, \infty)$.

Define

$$
z=x-\lambda^{*}
$$

Since $\lambda^{*}$ is a constant, we have

$$
\dot{z}=\dot{x}
$$

Design the upper bound $k_{b}$ and the lower bound $-k_{a}$ of $z$-state system as

$$
\begin{aligned}
& k_{a}=k_{c}-x(0), \\
& k_{b}=k_{c}-\lambda^{*},
\end{aligned}
$$

where $k_{c}$ is the upper bound of the light slip region. $x(0)$ is the initial state of the system (14). And the initial condition for the aircraft landing system $x(0)=0$. Since $k_{c}>\lambda^{*}$, we have the initial condition $z(0)=-\lambda^{*} \in\left(-k_{a}, k_{b}\right)$. And the initial condition of Lemma 2 is satisfied.

Choose an ABLF as

$$
\begin{aligned}
V(z)= & \frac{1}{2} q(z) \log \frac{k_{b}^{2}}{k_{b}^{2}-z^{2}} \\
& +\frac{1}{2}(1-q(z)) \log \frac{k_{a}^{2}}{k_{a}^{2}-z^{2}}
\end{aligned}
$$

where

$$
q(z)= \begin{cases}1, & \text { if } z>0 \\ 0, & \text { if } z \leq 0\end{cases}
$$

where $\log (\cdot)$ denotes the natural logarithm of $\cdot$. And for any $z \in\left(-k_{a}, k_{b}\right), V(z) \geq 0$. If and only if $z(t)=0$, we have $V(z)=0$. It implies that $V(z)$ is positive definite, since $\dot{V}(z)$ is continuous within each of the two intervals $z \in\left(-k_{a}, 0\right)$ and $z \in\left(0, k_{b}\right)$, respectively. Meanwhile, we have

$$
\lim _{z \rightarrow 0^{-}} \dot{V}(z)=\lim _{z \rightarrow 0^{+}} \dot{V}(z)=\dot{V}(0)=0 .
$$

It implies that $V(z)$ is continuously differentiable.

When $z>0, q(z)=1$. And $V(z)=\log \left(k_{b}^{2} /\left(k_{b}^{2}-z^{2}\right)\right)$. The time derivative of $V(z)$ is given by

$$
\dot{V}(z)=\frac{z \dot{z}}{k_{b}^{2}-z^{2}}=\frac{z\left(\dot{x}-\dot{\lambda}^{*}\right)}{k_{b}^{2}-z^{2}}=\frac{z(f(x)+g(x) u)}{k_{b}^{2}-z^{2}} .
$$

Design the control law $u$ as

$$
u=\frac{1}{g(x)}\left(-f(x)-k z\left(k_{b}^{2}-z^{2}\right)\right)
$$

where $k$ is a positive constant. Substituting (22) into (21), we obtain

$$
\dot{V}(z)=\frac{z(f(x)+g(x) u)}{k_{b}^{2}-z^{2}}=-k z^{2} .
$$

Since $k>0, \dot{V}(z)$ is negative definite for $\mathscr{D}_{1}: z>0$.

When $z \leq 0, q(z)=0$. The Lyapunov function $V(z)=$ $\log \left(k_{a}^{2} /\left(k_{a}^{2}-z^{2}\right)\right)$. Its derivative is

$$
\dot{V}(z)=\frac{z \dot{z}}{k_{a}^{2}-z^{2}}=\frac{z(f(x)+g(x) u)}{k_{a}^{2}-z^{2}} .
$$

Design the control law $u$ as

$$
u=\frac{1}{g(x)}\left(-f(x)-k z\left(k_{a}^{2}-z^{2}\right)\right)
$$

where $k$ is a positive constant. Substituting (25) into (24), we obtain

$$
\dot{V}(z)=\frac{z(f(x)+g(x) u)}{k_{a}^{2}-z^{2}}=-k z^{2} .
$$

Since $k>0, \dot{V}(z)$ is negative definite for $\mathscr{D}_{2}: z \leq 0$.

According to (22) and (25), the controller is designed as

$$
\begin{aligned}
u= & \frac{1}{g(x)}\{-f(x) \\
& \left.-k z\left[q(z)\left(k_{b}^{2}-z^{2}\right)+(1-q(z))\left(k_{a}^{2}-z^{2}\right)\right]\right\},
\end{aligned}
$$

where $q(z)$ is defined in (19).

According to (18) and (27), the derivative of the Lyapunov Function is

$$
\begin{aligned}
\dot{V}(z)= & q(z) \frac{z \dot{z}}{k_{b}^{2}-z^{2}}+(1-q(z)) \frac{z \dot{z}}{k_{a}^{2}-z^{2}} \\
= & q(z) \frac{z \dot{x}}{k_{b}^{2}-z^{2}}+(1-q(z)) \frac{z \dot{x}}{k_{a}^{2}-z^{2}} \\
= & q(z) \frac{z(f(x)+g(x) u)}{k_{b}^{2}-z^{2}} \\
& +(1-q(z)) \frac{z(f(x)+g(x) u)}{k_{a}^{2}-z^{2}}=-k z^{2} .
\end{aligned}
$$

$\dot{V}(z)$ is negative definite. The system is asymptotically stable. And $\lim _{t \rightarrow+\infty} x=\lambda^{*}$. Since the initial condition of Lemma 2 is satisfied, based on Lemma $2, z(t)$ remains in the open set $\left(-k_{a}, k_{b}\right), \forall t \in[0, \infty)$. From $x(t)=z(t)+\lambda^{*}, x(t)$ remains in the open set $\left(\lambda^{*}-k_{c}, k_{c}\right), \forall t \in[0, \infty)$. Thus, the output constraint will never be violated and the ABS will operate in the healthy region and the light slip region. 


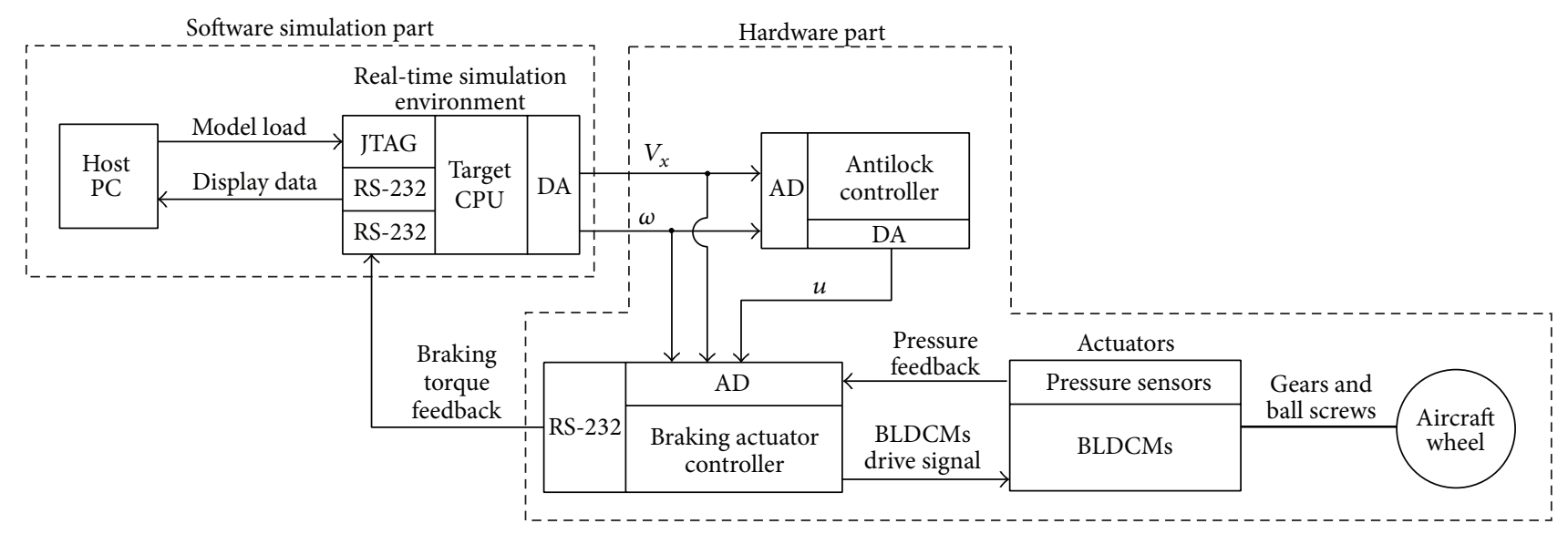

FIGURE 4: Structure of HIL experimental platform.

According to (27), $u$ is continuous within each of the two intervals $z \in\left(-k_{a}, 0\right)$ and $z \in\left(0, k_{b}\right)$, respectively. And there exists

$$
\lim _{z \rightarrow 0^{-}} \dot{u}=\lim _{z \rightarrow 0^{+}} \dot{u}=\dot{u}(0)=-\frac{f(x)}{g(x)} .
$$

It implies that $u$ is continuous and there is no chatter in the switching process.

\section{Hardware-In-Loop (HIL) Experiment}

In this section, a HIL experiment platform is established and HIL experiment results are addressed.

5.1. HIL Experiment Platform Setup. To verify the performance of the controller, the aircraft braking Hardware-InLoop (HIL) experimental platform is built and its structure is shown in Figure 4. The platform consisted of the host PC, the target CPU, the antilock controller, the braking actuator controller, the actuators, and the aircraft wheel. The host PC and the target CPU are the software simulation part, while the other four parts are hardware part.

Based on MATLAB/Simulink, the aircraft landing model, including the force balance model (described as (1) and (2)), the aircraft wheel model (described as (5)), and $\mu$ - $\lambda$ model (described as (7)), is established in host PC. With the help of the discrete tool in MATLAB, the aircraft landing model is discretized with the step of $0.00002 \mathrm{~s}$. With the tools of automatic code generation in MATLAB RTW, we rebuild the discrete aircraft landing model into the real-time code and download the real-time code into the target CPU through the JTAG. As the real-time simulation environment, the DSP TMS320F28335-based target CPU (shown in Figure 5(a)) sends the display data to host PC through RS-232 and provides the aircraft speed $V_{x}$ and the wheel speed $\omega$ to the antilock controller, which can be measured in practice by speed sensors. Based on DSP TMS320F28335, the antilock controller (shown in Figure 5(b)), which is the focus of this paper, is designed to give birth to the braking torque reference $u$. The detailed control strategy of the antilock controller is described in the last section. The braking actuator controller, which consisted of a DSP TMS320F2812-based CPU and a three-phase full-bridge power circuit (shown in Figure 5(c)), has a function to control the brushless DC motors (BLDCMs) of the actuators on the aircraft wheel such that the braking torque generated by the wheel asymptotically tracked the braking torque reference $u$ provided by the antilock controller. In detail, with the help of the gears and ball screws, BLDCMs exert pressures on the brake pads of the wheel and the mutual sliding between the brake pads gives birth to the braking torque. Since the braking torque is the proportional function related to the pressures provided by the BLDCMs, the braking torque feedback is observed by the proportional function related to the pressures which are measured by the pressure sensors. The actuator and the aircraft wheel are shown in Figure 5(d), and the picture of the platform is shown in Figure 5(e).

5.2. HIL Experiment Results. On the HIL experiment platform, we in this subsection conduct the ABLF-based antilock control experiment compared with the pressure-bias-modulated (PBM) technique [26], which is a well-known traditional aircraft antilock approach. The parameters of the aircraft landing system model are described by Tables 1 and 2. And the parameters of the wheel model are $B=0.01$, $R_{g}=0.3 \mathrm{~m}$, and $I=12 \mathrm{~kg} \cdot \mathrm{m}^{2}$. The limitation of the maximum braking torque is set at $20000 \mathrm{Nm}$. The initial conditions of the aircraft speed and the wheel speed are $V_{x}(0)=$ $72 \mathrm{~m} / \mathrm{s}$ and $R_{g} \omega(0)=72 \mathrm{~m} / \mathrm{s}$, respectively. This implies that $\lambda(0)=0$. And the lower bound of the aircraft speed is set as $0.1 \mathrm{~m} / \mathrm{s}$. Once the aircraft speed $V_{x}$ is lower than this lower bound speed, ABS will be turned off. Correspondingly, the aircraft stops by itself. In the $\mu$ - $\lambda$ function, the optimum wheel slip ratio $\lambda^{*}=0.25$ and the maximum friction coefficient $\mu^{*}=0.6$. The parameters of the antilock controller are chosen as follows: $k=100, k_{a}=0.3$, and $k_{b}=0.05$.

The experimental results of the proposed ABLF-based antilock control and PBM control are shown in Figures 611. From these experimental results in Figures 6-11, it can be clearly observed that under the proposed ABLF-based 


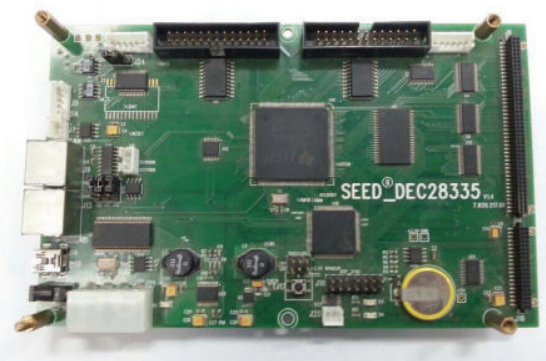

(a) Target CPU

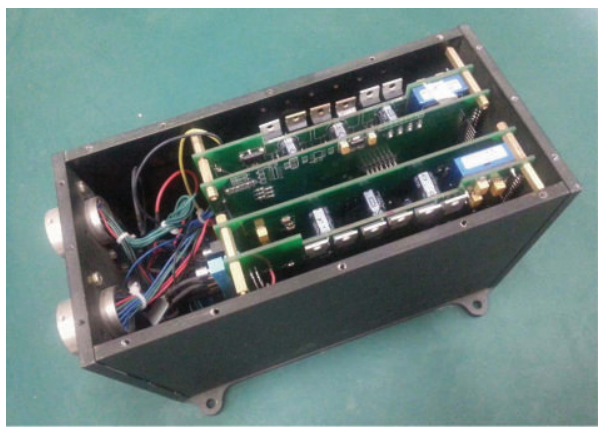

(c) Braking actuator controller

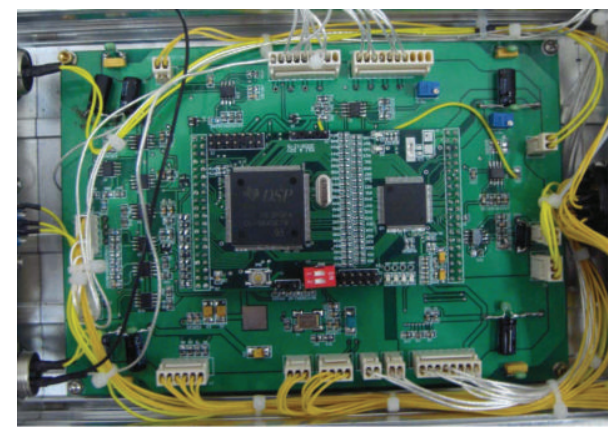

(b) Antilock controller

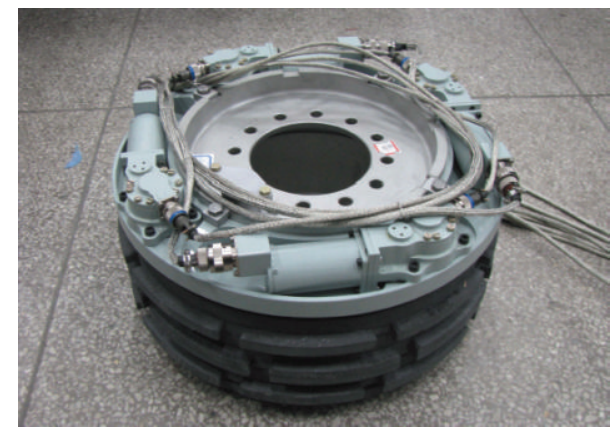

(d) Actuator and aircraft wheel

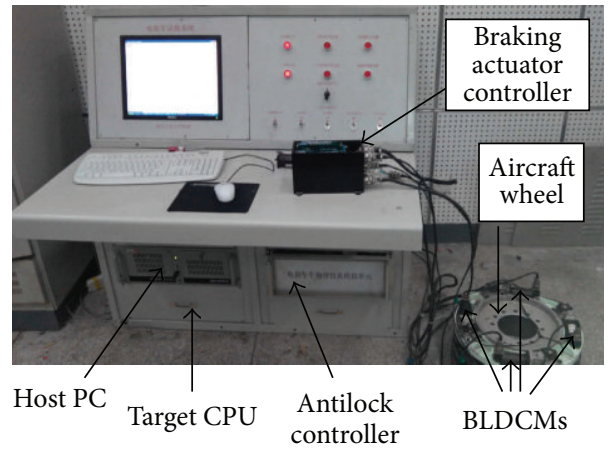

(e) HIL experimental platform picture

FIGURE 5: Hardware-In-Loop experimental platform.

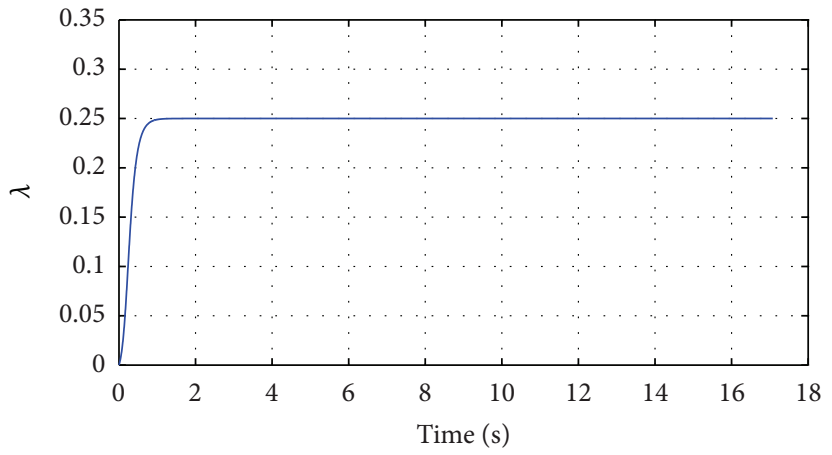

(a) Antilock control based on ABLF

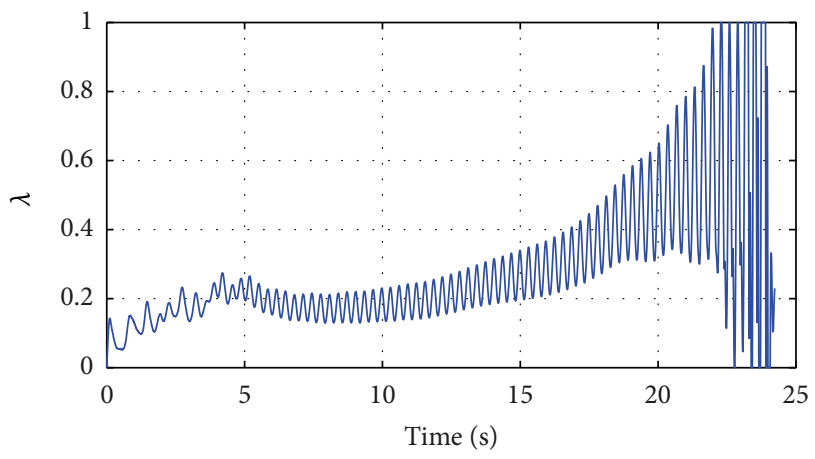

(b) PBM control

FIgURE 6: Wheel slip ratio $\lambda$. 


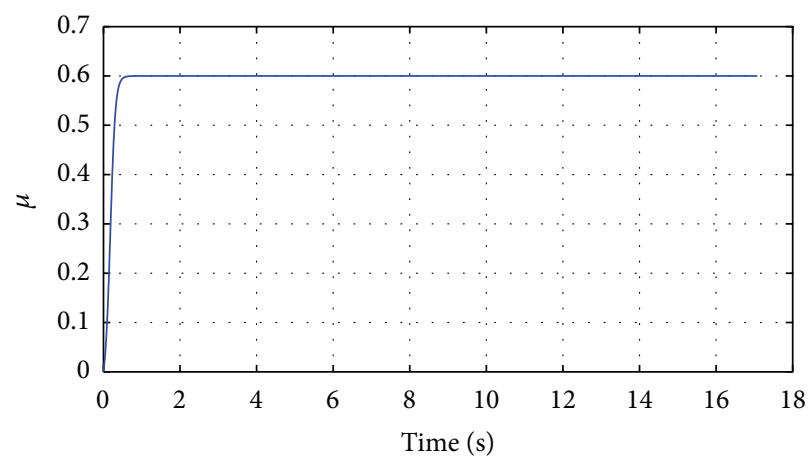

(a) Antilock control based on ABLF

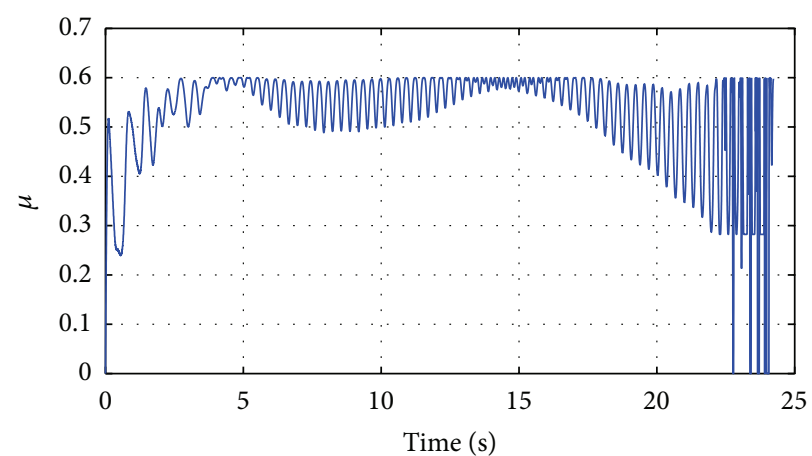

(b) PBM control

FIGURE 7: Friction coefficient $\mu$.

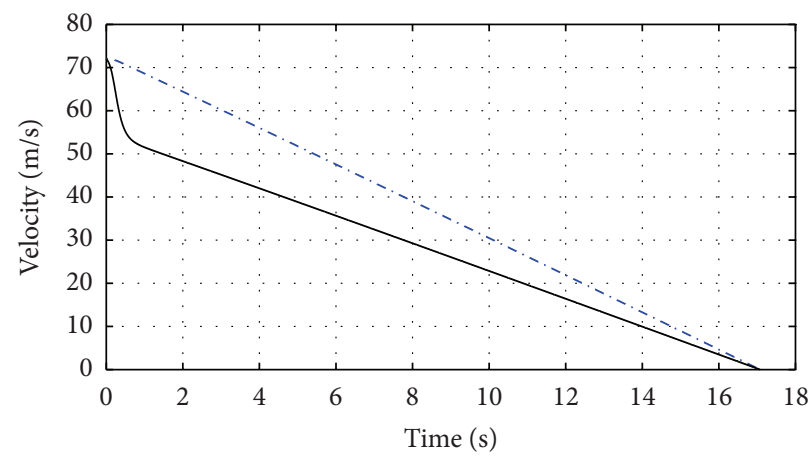

(a) Antilock control based on ABLF

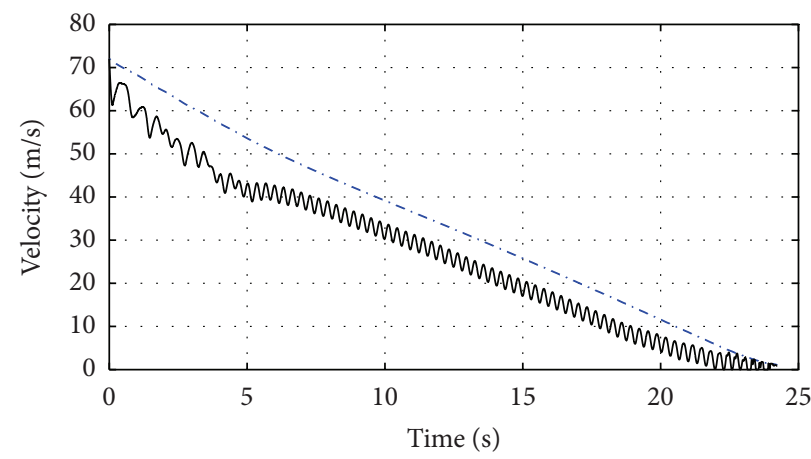

(b) PBM control

Figure 8: Aircraft speed and wheel speed.

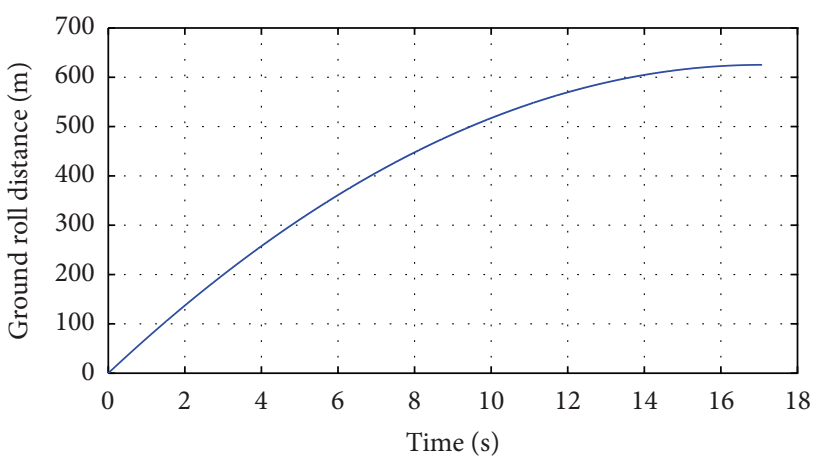

(a) Antilock control based on ABLF

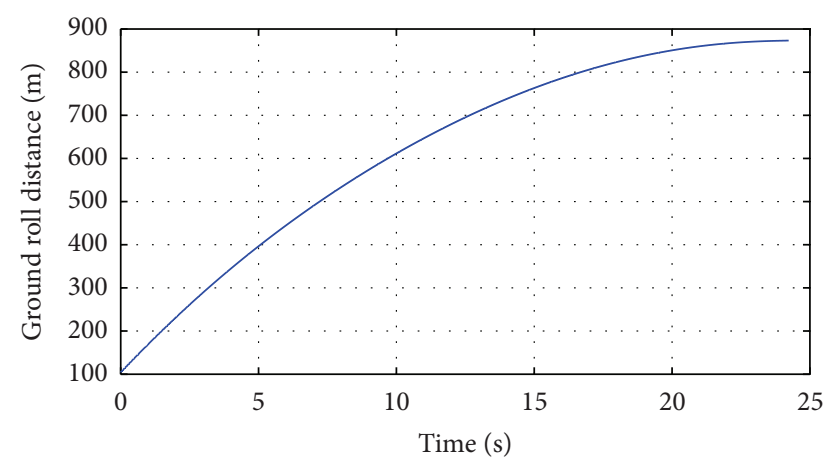

(b) PBM control

FIgURE 9: Stopping distance.

antilock control the wheel slip ratio tracks the optimal slip ratio with zero steady state error after a short time transient. And in the whole braking process, the ABS operates in the healthy region initially and then at the optimal braking status. This implies that the self-locking of the aircraft wheel is avoided completely. While under PBM control, the wheel slip ratio cannot track the optimal slip ratio well and it finally works in deep slip region where self-locking will occur. Correspondingly, compared with PBM control, the ABLFbased antilock control proposed has a better performance in wheel slip ratio, friction coefficient, wheel speed, stopping time, stopping distance, and braking torque.

For instance, Figure 6 shows the wheel slip ratio $\lambda$. Under ABLF-based antilock control, after a short time transient (less than $1 \mathrm{~s}$ ), the wheel slip ratio tracks optimal slip ratio 0.25 without steady state error, while under PBM control, its slip ratio is around optimal slip ratio 0.25 when the aircraft speed is in the middle speed process. Yet the slip ratio has an obvious tracking error when the aircraft is in the high or low speed process. Figure 7 shows the friction 
TABLE 3: The performance comparison of antilock control based on ABLF and PBM control.

Comparison items Antilock control based on ABLF PBM control

Tracking the optimum slip ratio without steady state error

Tracking the maximum friction coefficient without steady state error

Stopping time

\begin{tabular}{cc} 
Yes & No \\
Yes & No \\
$17 \mathrm{~s}$ & $24.4 \mathrm{~s}$ \\
$625 \mathrm{~m}$ & $885 \mathrm{~m}$ \\
Yes & No \\
\hline
\end{tabular}

$\begin{array}{cc}\text { Yes } & \text { No } \\ \text { Yes } & \text { No } \\ 17 \mathrm{~s} & 24.4 \mathrm{~s} \\ 625 \mathrm{~m} & 885 \mathrm{~m} \\ \text { Yes } & \text { No }\end{array}$

Stopping distance

Only operating in the healthy region and the light slip region

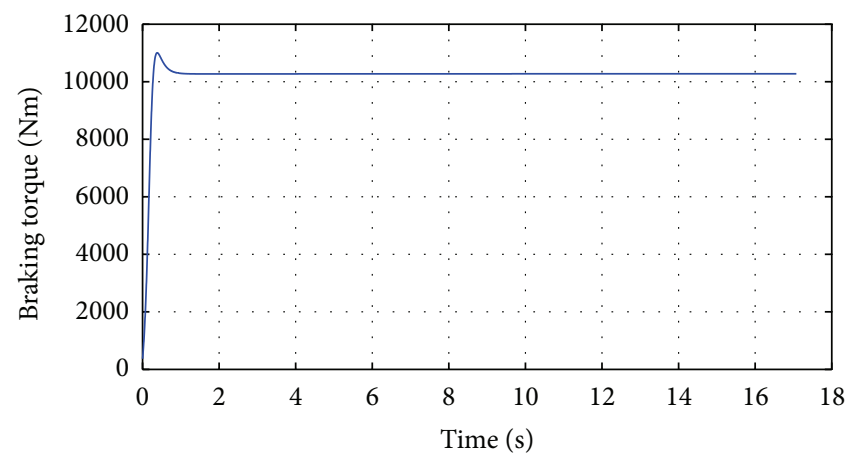

(a) Antilock control based on ABLF

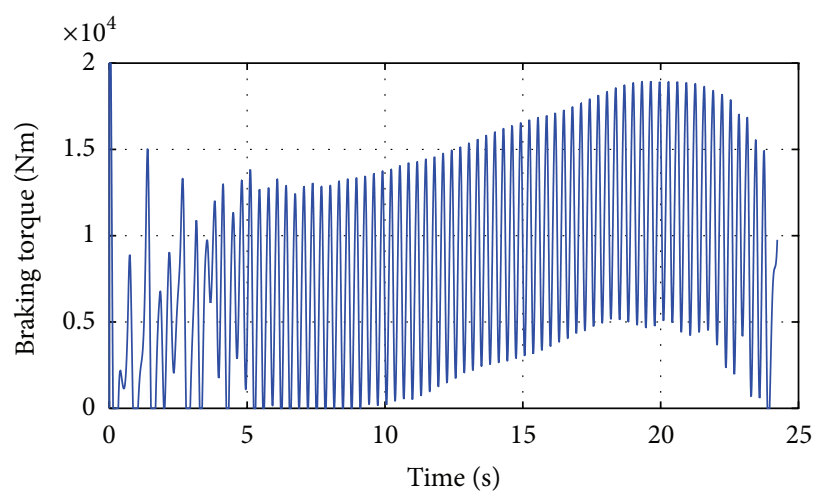

(b) PBM control

FIgURE 10: Braking torque.

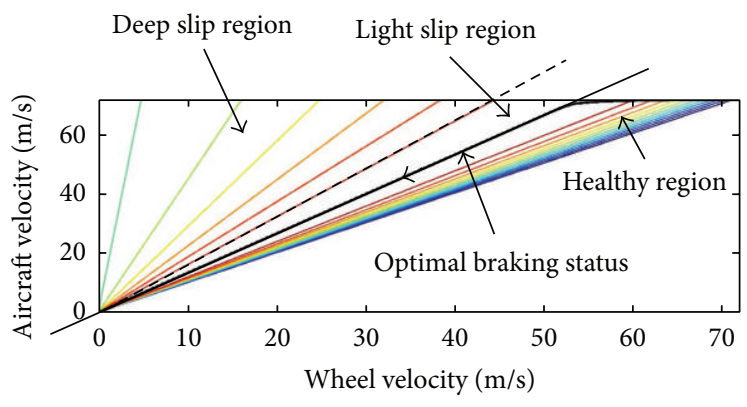

(a) Antilock control based on ABLF

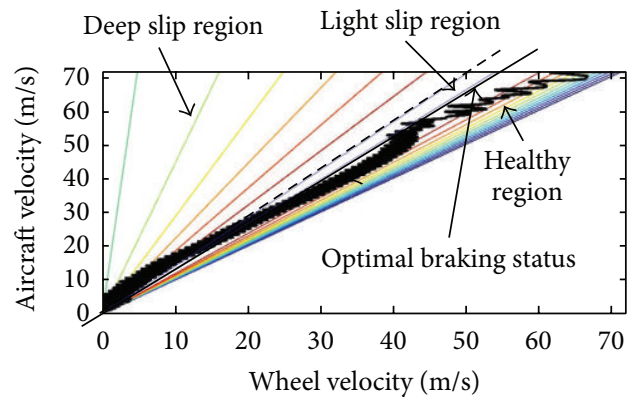

(b) PBM control

FIGURE 11: Contour of friction coefficient.

coefficient $\mu$. Under ABLF-based antilock control, after a short time transient process (also less than $1 \mathrm{~s}$ ), the friction coefficient follows the maximum friction coefficient 0.6 with zero steady state error, which implies the ABS will provide the maximum braking force at its steady state, while under PBM control, only when the ABS works in the middle speed process, the friction coefficient is around the maximum friction coefficient 0.6; when the ABS works at the high or low speed process, the friction coefficient will deviate from the maximum friction coefficient. Figure 8 shows the aircraft speed $V_{x}$ and the wheel speed $\omega R_{g}$. Compared with the PBM control, the wheel speed of the ABLF-based antilock control has no oscillation. Figure 9 shows the stopping distance. The performance of the stopping time and the stopping distance under the ABLF-based antilock control $(17 \mathrm{~s}$ and $625 \mathrm{~m}$, resp.) is also better than the one under PBM control $(24.4 \mathrm{~s}$ and $885 \mathrm{~m}$, resp.). Figure 10 shows the braking torque $T_{b}$. Compared with PBM control, the braking torque of the
ABLF-based antilock control has no oscillation which is good at extending the life of actuator.

Figure 11 describes the contour of friction coefficient. It can be observed clearly that the braking operating region is divided into three regions (the healthy region, the light slip region, and the deep slip region) by the dash lines. Under the ABLF-based antilock control, the bold solid line is the actual braking status. It is in the healthy region firstly and then it is at the optical braking status. Compared with the control scheme proposed, the braking operating status of PBM control is in deep slip region at the braking low speed process, which will cause wheel self-locking. Based on the HIL experiment results, the performance comparison of the two control schemes is provided in Table 3.

\section{Conclusion}

In this paper, aircraft braking operating status is divided into three regions, which reflect the wheel slip degree directly. An 
ABLF-based wheel slip controller is proposed, which not only implements zero steady state error tracking of the wheel slip ratio but also constrains the braking system operating in the healthy region and the light slip region. And in contrast to existing antilock control method, it prevents wheels from selflocking completely.

\section{Conflict of Interests}

The authors declare that there is no conflict of interests regarding the publication of this paper.

\section{Acknowledgments}

This work was supported in part by the NSF of China under Grant 51407143 and by Specialized Research Fund for the Doctoral Program of Higher Education under Grant 20136102120049.

\section{References}

[1] B. Subudhi and S. S. Ge, "Sliding-mode-observer-based adaptive slip ratio control for electric and hybrid vehicles," IEEE Transactions on Intelligent Transportation Systems, vol. 13, no. 4, pp. 1617-1626, 2012.

[2] C.-M. Lin and C.-F. Hsu, "Self-learning fuzzy sliding-mode control for antilock braking systems," IEEE Transactions on Control Systems Technology, vol. 11, no. 2, pp. 273-278, 2003.

[3] V. Ćirović and D. Aleksendrić, "Adaptive neuro-fuzzy wheel slip control," Expert Systems with Applications, vol. 40, no. 13, pp. 5197-5209, 2013.

[4] A. Harifi, A. Aghagolzadeh, G. Alizadeh, and M. Sadeghi, "Designing a sliding mode controller for slip control of antilock brake systems," Transportation Research Part C: Emerging Technologies, vol. 16, no. 6, pp. 731-741, 2008.

[5] B.-C. Chen and H. Peng, "Differential-braking-based rollover prevention for sport utility vehicles with human-in-the-loop evaluations," Vehicle System Dynamics, vol. 36, no. 4-5, pp. 359$389,2001$.

[6] R. De Castro, R. E. Araujo, and D. Freitas, "Wheel slip control of EVs based on sliding mode technique with conditional integrators," IEEE Transactions on Industrial Electronics, vol. 60, no. 8, pp. 3256-3271, 2013.

[7] H. Mirzaeinejad and M. Mirzaei, "A novel method for nonlinear control of wheel slip in anti-lock braking systems," Control Engineering Practice, vol. 18, no. 8, pp. 918-926, 2010.

[8] W.-C. Lin, C.-L. Lin, P.-M. Hsu, and M.-T. Wu, "Realization of anti-lock braking strategy for electric scooters," IEEE Transactions on Industrial Electronics, vol. 61, no. 6, pp. 2826-2833, 2014.

[9] D. Capra, E. Galvagno, V. Ondrak, B. van Leeuwen, and A. Vigliani, "An ABS control logic based on wheel force measurement," Vehicle System Dynamics, vol. 50, no. 12, pp. 1779-1796, 2012.

[10] J. Yi, L. Alvarez, and R. Horowitz, "Adaptive emergency braking control with underestimation of friction coefficient," IEEE Transactions on Control Systems Technology, vol. 10, no. 3, pp. 381-392, 2002.
[11] C. Mi, H. Lin, and Y. Zhang, "Iterative learning control of antilock braking of electric and hybrid vehicles," IEEE Transactions on Vehicular Technology, vol. 54, no. 2, pp. 486-494, 2005.

[12] A. Mirzaei, M. Moallem, B. Mirzaeian, and B. Fahimi, "Design of an optimal fuzzy controller for antilock braking systems," in Proceedings of the IEEE Conference on Vehicle Power and Propulsion, pp. 823-828, September 2005.

[13] J. R. Layne, K. M. Passino, and S. Yurkovich, "Fuzzy learning control for antiskid braking systems," IEEE Transactions on Control Systems Technology, vol. 1, no. 2, pp. 122-129, 1993.

[14] A. Badie Sharkawy, "Genetic fuzzy self-tuning PID controllers for antilock braking systems," Engineering Applications of Artificial Intelligence, vol. 23, no. 7, pp. 1041-1052, 2010.

[15] S. Mahanty and S. C. Subramanian, "A non-linear model-based slip controller for electropneumatic brakes in heavy commercial vehicles," International Journal of Heavy Vehicle Systems, vol. 20, no. 1, pp. 35-60, 2013.

[16] M. Tanelli, A. Astolfi, and S. M. Savaresi, "Robust nonlinear output feedback control for brake by wire control systems," Automatica, vol. 44, no. 4, pp. 1078-1087, 2008.

[17] C. Zhang and R. Ordóñez, "Numerical optimization-based extremum seeking control with application to ABS design," IEEE Transactions on Automatic Control, vol. 52, no. 3, pp. 454467, 2007.

[18] E. Dinçmen, B. A. Güvenç, and T. Acarman, "Extremum-seeking control of ABS braking in road vehicles with lateral force improvement," IEEE Transactions on Control Systems Technology, vol. 22, no. 1, pp. 230-237, 2014.

[19] E. Dinçmen and B. A. Güvenç, "A control strategy for parallel hybrid electric vehicles based on extremum seeking," Vehicle System Dynamics, vol. 50, no. 2, pp. 199-227, 2012.

[20] P. Wellstead and N. Pettit, "Analysis and redesign of an antilock brake system controller," IEE Proceedings-Control Theory and Applications, vol. 144, no. 5, pp. 413-426, 1997.

[21] K. P. Tee, S. S. Ge, and F. E. H. Tay, "Adaptive control of electrostatic microactuators with bidirectional drive," IEEE Transactions on Control Systems Technology, vol. 17, no. 2, pp. 340-352, 2009.

[22] K. P. Tee and S. S. Ge, "Control of nonlinear systems with partial state constraints using a barrier lyapunov function," International Journal of Control, vol. 84, no. 12, pp. 2008-2023, 2011.

[23] K. P. Tee, B. Ren, and S. S. Ge, "Control of nonlinear systems with time-varying output constraints," Automatica, vol. 47, no. 11, pp. 2511-2516, 2011.

[24] K. P. Tee, S. S. Ge, and E. H. Tay, "Barrier lyapunov functions for the control of output-constrained nonlinear systems," Automatica, vol. 45, no. 4, pp. 918-927, 2009.

[25] K. B. Ariyur and M. Krstić, Real-Time Optimization by Extremum-Seeking Control, John Wiley \& Sons, 2003.

[26] Z. Ming, N. Hong, W. Xiao-Hui, and Z. Enzhi, "Research on modelling and simulation for aircraft anti-skid braking," in Proceedings of the 2nd International Symposium on Systems and Control in Aerospace and Astronautics (ISSCAA '08), pp. 1-5, IEEE, Shenzhen, China, December 2008. 

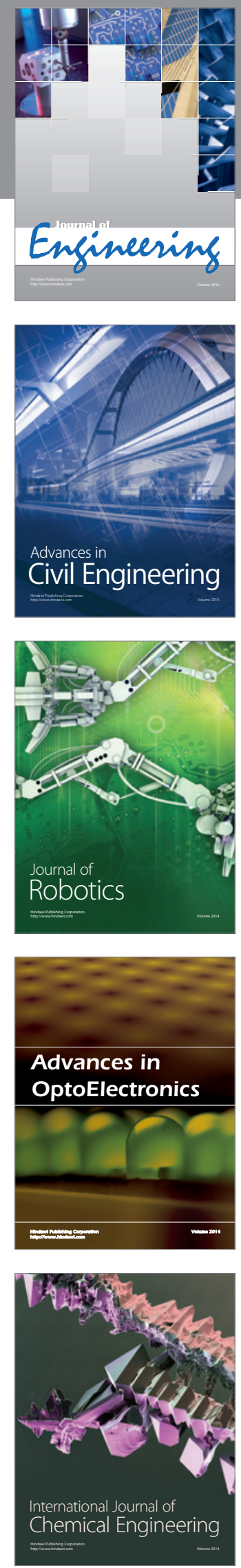

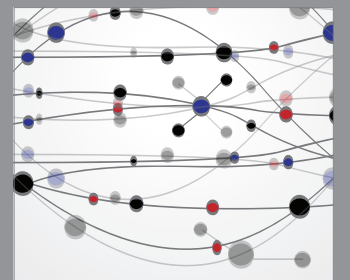

The Scientific World Journal
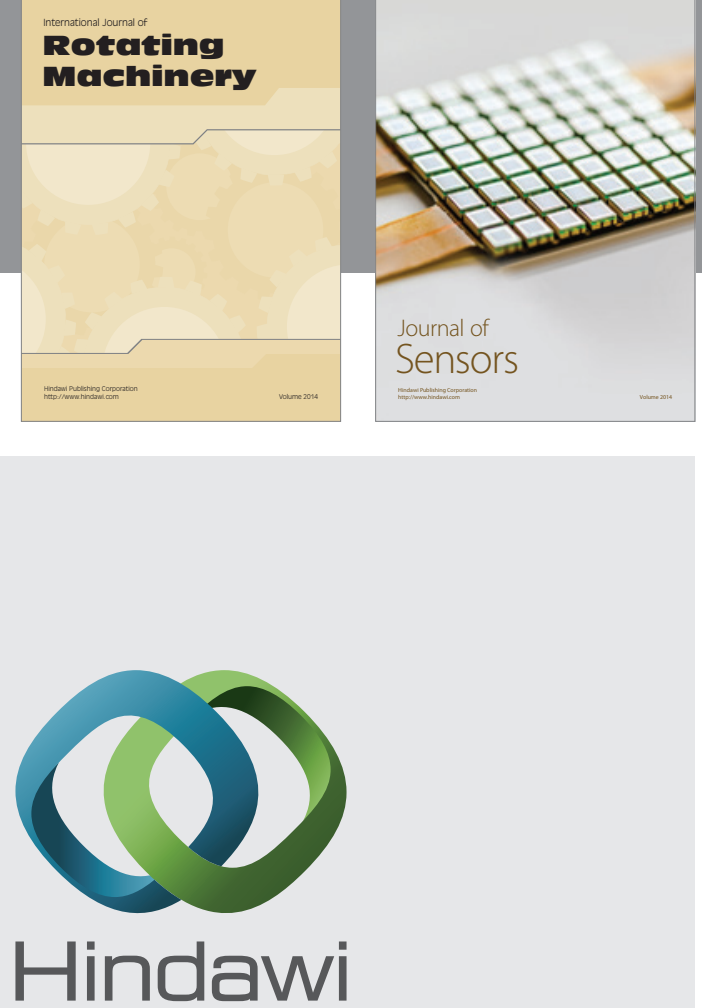

Submit your manuscripts at http://www.hindawi.com
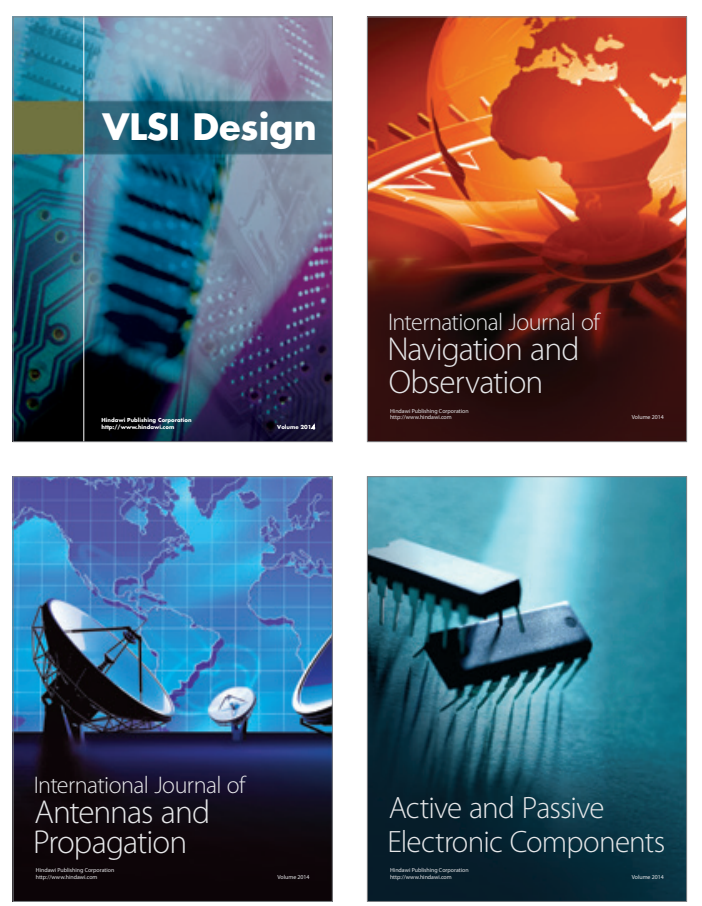
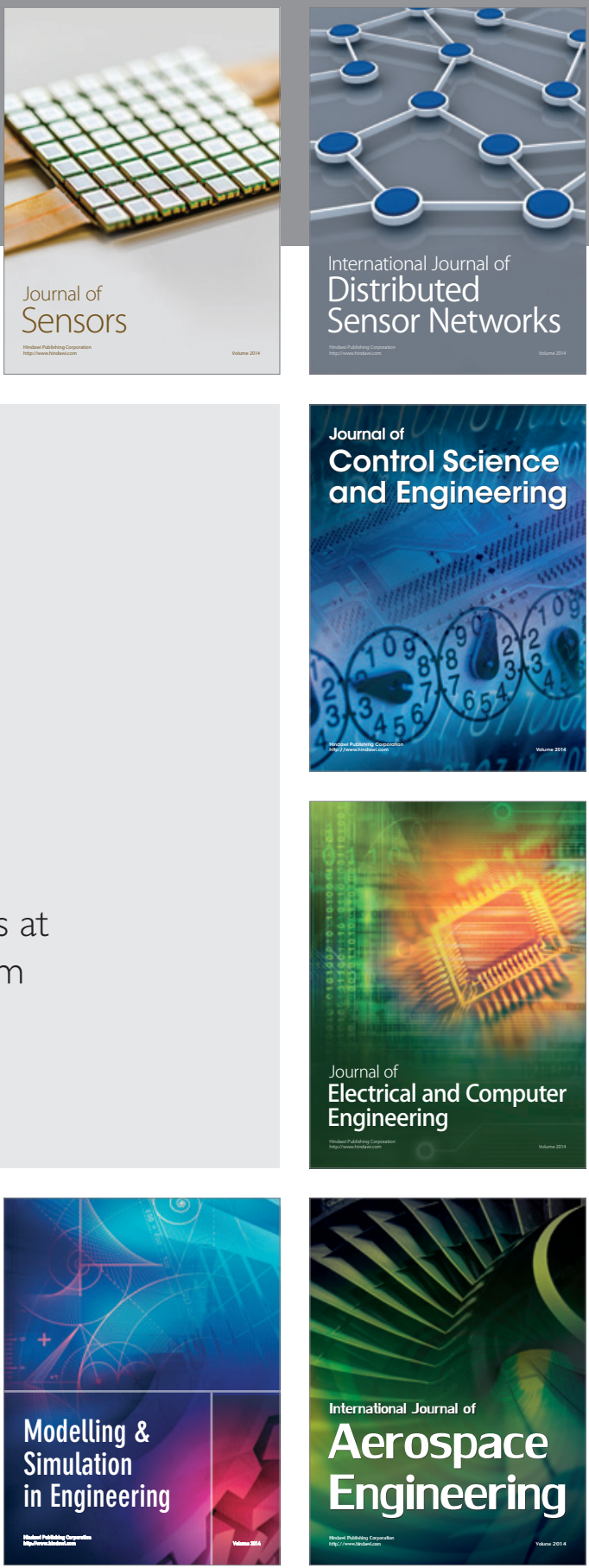

Journal of

Control Science

and Engineering
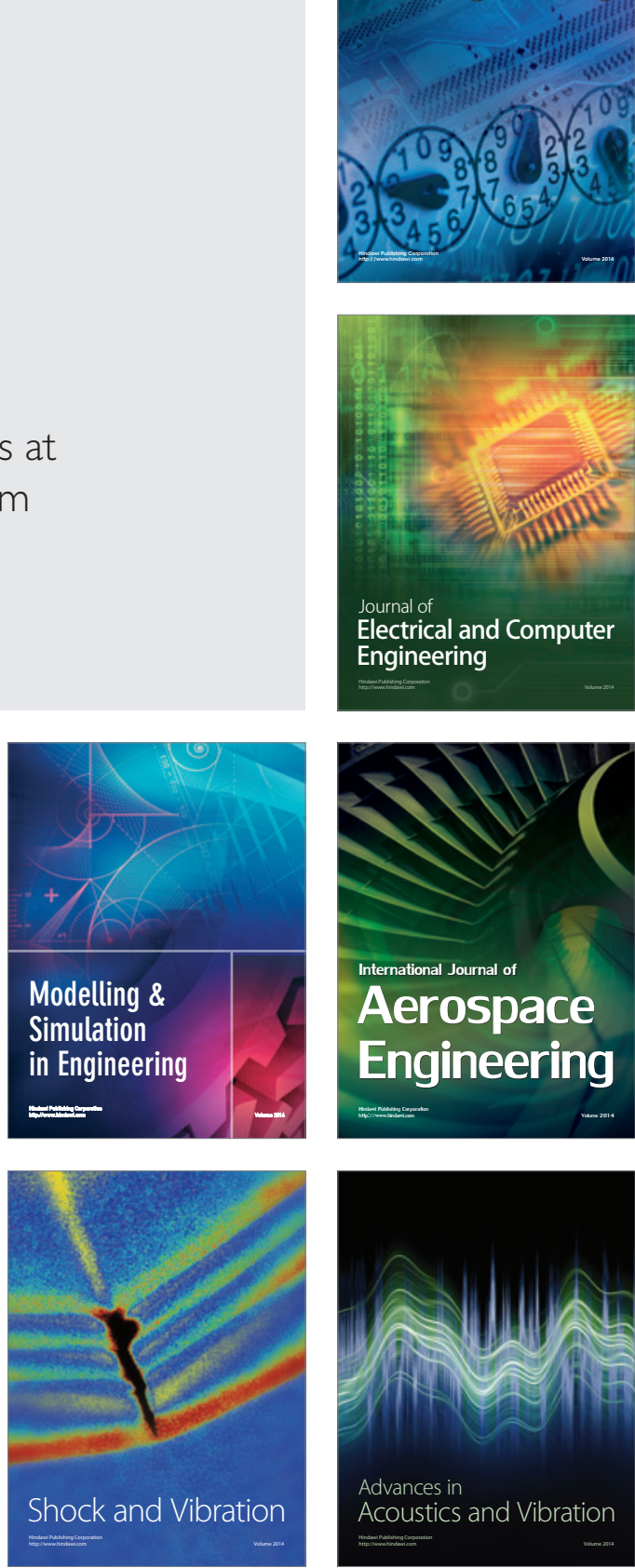